\section{Patterns of progression of localized retinal nerve fibre layer defect on red-free fundus photographs in normal-tension glaucoma}

MH Suh"1, DM Kim¹, YK Kim¹, T-W Kim²

and $\mathrm{KH}$ Park $^{1}$
Eye (2010) 24, 857-863; doi:10.1038/eye.2009.209;

published online 14 August 2009

Keywords: localized retinal nerve fibre layer defect; pattern of progression of defect; normaltension glaucoma

\section{Introduction}

Retinal nerve fibre layer (RNFL) defect is one of the most sensitive parameters in detecting glaucomatous optic neuropathy, ${ }^{1}$ and serial RNFL examinations by red-free fundus photography are known to be more sensitive than colour disc or visual field evaluations in detecting progression of glaucomatous optic neuropathies. ${ }^{2,3}$ For all recent advances in ocular imaging such as scanning laser polarimetry and optical coherence tomography, detection of RNFL defect progression is hampered, in part, by rapidly evolving changes in technology and variability of measurements. ${ }^{4,5}$ Therefore, red-free fundus photography still remains a useful tool in detecting progression of RNFL defect.

An earlier study evaluated RNFL defect progression in patients with ocular hypertension, ${ }^{6}$ but little is known about patterns of RNFL defect progression. In this study, we investigated patterns of localized RNFL defect progression on serial red-free fundus photographs and quantified extents of progression in normal-tension glaucoma (NTG) patients.
1Department of Ophthalmology, Seoul National University Hospital, Seoul, Korea

${ }^{2}$ Department of Ophthalmology, Seoul National University Bundang Hospital, Seongnam, Korea

Correspondence: DM Kim, Department of Ophthalmology, Seoul National University Hospital, 28 Yongon-Dong, Chongno-Gu, Seoul 110-744, Korea

Tel: + 8222072 2087; Fax: +8227413187. E-mail:dmkim@ snu.ac.kr

Received: 24 March 2009 Accepted in revised form: 7 July 2009

Published online: 14 August 2009 


\section{Materials and methods}

Consecutive 79 eyes of 71 NTG patients that had shown progression of localized RNFL defect on serial red-free fundus photographs were initially selected by one ophthalmologist (DMK). These patients were followed up at the Glaucoma Clinic of Seoul National University Hospital between January 2003 and December 2008. NTG was defined according to the following criteria: diurnal intraocular pressure (IOP) of $21 \mathrm{~mm} \mathrm{Hg}$ or lower without any medication, glaucomatous optic neuropathy, glaucomatous visual field defect, open anterior chamber angle, and no evidence of non-glaucomatous cause of optic nerve damage. Each subject completed visual acuity measurement, refraction, slit-lamp biomicroscopy, diurnal IOP measurements with Goldmann applanation tonometry, central corneal thickness measurement, gonioscopy, disc stereophotography, red-free fundus photography, and automated perimetry. When the result of Humphrey central 30-2 threshold visual field test was within normal limits $(n=16)$, Humphrey Matrix 30-2 visual field test was performed. Disc stereophotography, red-free fundus photography, and automated perimetry were performed every 1 to $11 / 2$ years. Disc stereophotograph and red-free fundus photograph were taken using a digital Kowa fundus camera (VX-10, Tokyo, Japan) after maximum pupil dilation by one technician. Sixty-degree, wide-angle views of the optic disc, carefully focused on the retina using the built-in split-line focusing device and centred between the fovea and the optic disc were obtained for red-free fundus photograph and reviewed on an LCD monitor. ${ }^{7}$ Localized RNFL defect was defined as a dark wedgeshaped area with its tip touching the optic disc border in the brightly striated pattern of the surrounding RNFL. ${ }^{8}$ The width of the defect at 1-disc diameter distance from the edge of the optic disc should be larger than that of major retinal vessel. ${ }^{8}$ The parameters used to represent localized RNFL defect (angles $\alpha$ and $\beta$ ) were defined as described earlier (Figure 1). ${ }^{9}$ Angle $\alpha$ is the minimum angle that is made by a line from the centre of the optic disc to the centre of the macula and a line from the centre of the optic disc to the optic disc margin where the nearest border of RNFL defect meets the optic disc. The smaller the angle, the closer the RNFL defect to the fovea and the closer the visual field defect to the fixation. Angle $\beta$ represents the circumferential angle of the RNFL defect. The larger the angle, the wider the RNFL defect in circumferential width and the more diffuse the visual field defect.

Two examiners (MHS, YKK) masked to the subjects' identities and test results classified 79 eyes into one of the following categories after reviewing serial red-free fundus photographs of them: widening or deepening of
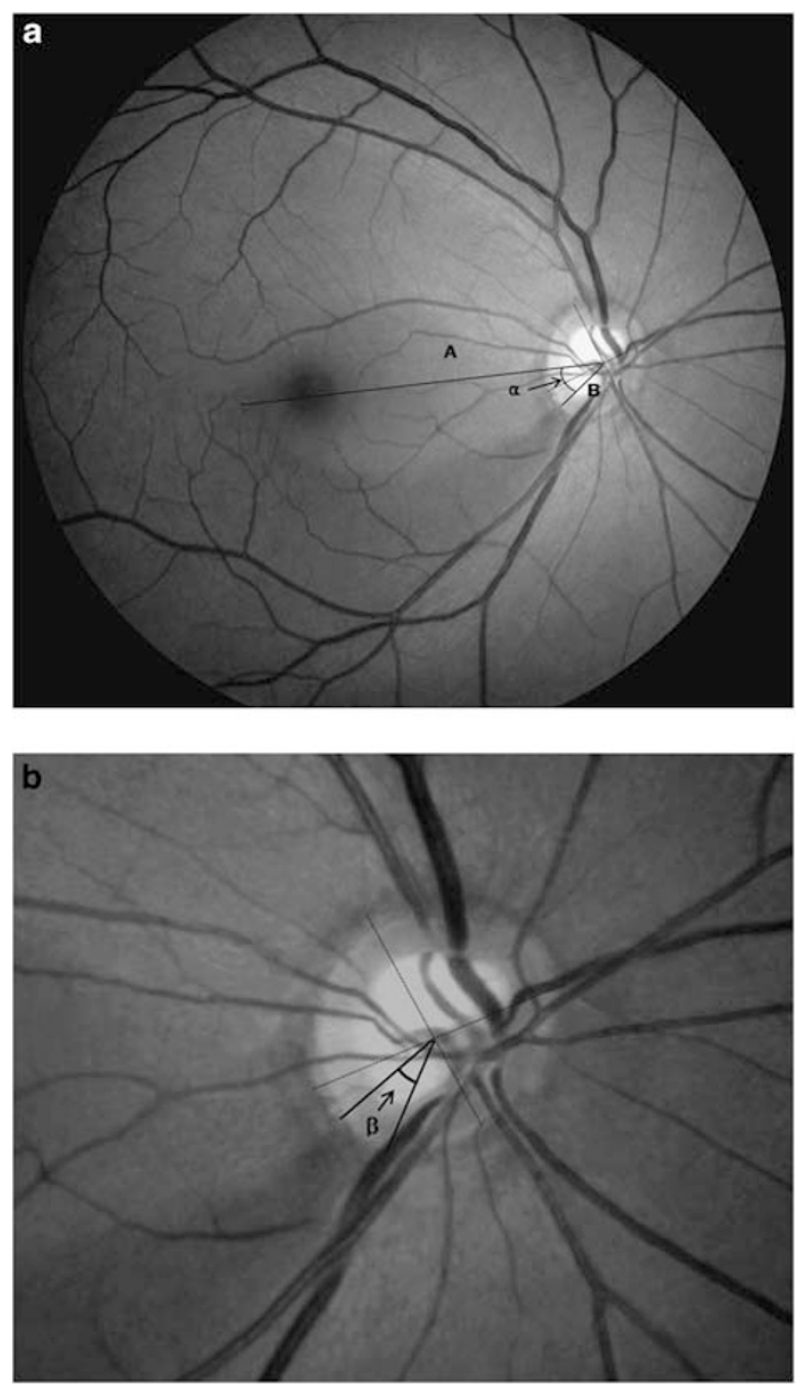

Figure 1 Parameters used to represent localized retinal nerve fibre layer (RNFL) defect. (a) Angle $\alpha$ is the minimum angle that is made by a line ' $A$ ' from the centre of the optic disc to the centre of the macula and a line ' $\mathrm{B}$ ' from the centre of the optic disc to the disc margin where the nearest border of RNFL defect meets the optic disc. (b) Angle $\beta$ represents the circumferential angle of the RNFL defect.

the pre-existing defect, appearance of a new defect, or an ambiguous one. Widening was defined as a recognizable increase in a distance between the border of the RNFL defect and the adjacent reference vessels. In these cases, the difference of angle $\beta$ and the direction of the widening (towards or away from the macula) were determined using the angle-measuring program (Maro-view 5.4, Infinitt, Seoul, Korea) on an LCD monitor. Depth of the RNFL defect was determined according to the following two methods after equalizing brightness and contrast of RNFL at the posterior pole between two images on an LCD monitor. First, severity of the RNFL defect was divided into three grades (Grade 1: 
mild defect; Grade 2: moderate defect; Grade 3: severe defect) by evaluating brightness and texture of the RNFL and degree of blood vessel obscuration. This method was described in detail elsewhere. ${ }^{1}$ Grade 1 indicates fine striations of RNFL is visible and only large vessels are clearly visible; Grade 2, striations of RNFL defect is barely detectable and even small vessels are clearly visible; and Grade 3, texture of RNFL is not visible at all. ${ }^{1}$ Second, sharpness of temporal and nasal borders of the RNFL defect was compared between two images. Deepening was defined as increase in the grade of severity or as sharpening of the border of the pre-existing RNFL defect. Appearance of a new defect was determined when a localized defect ranging from Grades 1 to 3 newly developed in the area where there had been no defect. ${ }^{1}$ When the photograph had poor quality of image or a change was too subtle to be considered progression, we classified them as ambiguous one and excluded from further evaluation. Evaluation of red-free fundus photographs was independently performed twice in each examiner (MHS and YKK) and inter-observer discrepancies were resolved by adjudication of a third experienced examiner (DMK). Absolute values and changes of angles $\alpha$ and $\beta$ were determined by averaging values measured by two examiners. In case of bilateral progression, only one eye was randomly selected not to mislead baseline characteristics such as age, gender, follow-up period, and systemic diseases.

Serial assessments of disc stereophotographs and visual fields were also performed to detect progression. Progressive changes of the optic disc were defined as narrowing or notching of the neural rim, increased cup:disc ratio, or shift in the position of vasculature. The optic disc changes were also evaluated by the two examiners in the same manner as done in the evaluation of red-free fundus photographs and the discrepancy between them was resolved by adjudication of a third experienced examiner. Visual field defect progression was defined as $\geqslant 2$ locations of existing defects or those contiguous to them that have worsened by $\geqslant 10 \mathrm{~dB}$ in pattern deviation plots in at least two consecutive tests ${ }^{10}$ or as 'likely progression' according to the GPA program of Humphrey Field Analyser. In cases that showed progression on the visual fields, pattern of progression was divided into three categories: deepening or extension of the pre-existing scotoma or a new scotoma not within or adjacent to the existing scotoma. ${ }^{11}$ The study protocol was approved by the institutional review board of the Seoul National University Hospital.

\section{Results}

Among 79 eyes of 71 NTG patients enrolled, 10 eyes classified into ambiguous one and 4 eyes that had bilateral progression were excluded from evaluation. Therefore, 65 eyes of 65 patients were included. Demographics and characteristics of ophthalmic measurements are presented in Table 1. Mean age at diagnosis was $56.2 \pm 10.8$ years and female patients was $66.2 \%$. Mean follow-up period was $52.4 \pm 31.4$ months. Fifty and 65 patients were on medications before and at the time of progression, respectively. Numbers of medication were $1.4 \pm 0.1$ (range: $0-3$ ) and $1.9 \pm 0.6$ (range: 1-3), respectively. No patient had ocular surgery or laser treatment. Table 2 shows intra- and interobserver variability in determining progression of the RNFL defect. Both intra- and inter-observer agreement were good in each criterion of progression ( $\kappa$ values $\geqslant 0.79$ ) (Table 2).

Table 1 Demographics and characteristics of ophthalmic measurements in the eyes with progression of localized retinal nerve fibre layer defect $(n=65)$

\begin{tabular}{|c|c|}
\hline \multicolumn{2}{|l|}{ Variables } \\
\hline Age at diagnosis & $56.2 \pm 10.8$ years \\
\hline \multicolumn{2}{|l|}{ Age distribution } \\
\hline$\leqslant 45$ years & $16.9 \%$ \\
\hline $46-64$ years & $53.9 \%$ \\
\hline$\geqslant 65$ years & $29.2 \%$ \\
\hline Female & $66.2 \%$ \\
\hline Follow-up period & $52.4 \pm 31.4$ months \\
\hline \multicolumn{2}{|l|}{ Systemic diseases } \\
\hline Diabetic mellitus & $1.5 \%$ \\
\hline Systemic hypertension & $20.0 \%$ \\
\hline Central corneal thickness & $518.4 \pm 19.7 \mu \mathrm{m}$ \\
\hline $\begin{array}{l}\text { Highest intraocular pressure at diurnal } \\
\text { intraocular pressure measurements }\end{array}$ & $14.3 \pm 1.9 \mathrm{~mm} \mathrm{Hg}$ \\
\hline \multicolumn{2}{|l|}{ Location of localized retinal nerve fibre layer defect } \\
\hline $\begin{array}{l}\text { Superotemporal retina/inferotemporal } \\
\text { retina/both }\end{array}$ & $5 / 50 / 10$ eyes \\
\hline \multicolumn{2}{|l|}{$\begin{array}{l}\text { Parameters of Humphrey central 30-2 threshold } \\
\text { visual field at initial visit }(n=49)\end{array}$} \\
\hline Mean deviation & $-3.9 \pm 2.9 \mathrm{~dB}$ \\
\hline Pattern standard deviation & $6.5 \pm 3.9 \mathrm{~dB}$ \\
\hline \multicolumn{2}{|c|}{$\begin{array}{l}\text { Parameters of Humphrey Matrix 30-2 visual field at } \\
\text { initial visit }(n=16)\end{array}$} \\
\hline Mean deviation & $-4.0 \pm 4.5 \mathrm{~dB}$ \\
\hline Pattern standard deviation & $3.9 \pm 0.9 \mathrm{~dB}$ \\
\hline \multicolumn{2}{|l|}{ Cup:disc ratio at initial visit } \\
\hline Horizontal & $0.5 \pm 0.2$ \\
\hline Vertical & $0.6 \pm 0.2$ \\
\hline
\end{tabular}

$\mathrm{dB}$, decibels.

Data are provided as mean \pm SD unless otherwise indicated. 
Most of localized RNFL defects located at the inferotemporal retina $(n=50)$. The most common pattern of progression of localized RNFL defect was widening of the defect towards the macula $(n=37 ; 56.9 \%)$ (Figure 2a), and this was followed by deepening of the defect $(n=25$; $38.5 \%$ ) (Figure 3a and b), appearance of a new defect $(n=6 ; 9.2 \%)$ (Figure 4$)$, and widening of the defect away from the macula $(n=5 ; 7.7 \%)$ (Figure $2 b$ ). Eight eyes simultaneously showed two patterns of progression. Three eyes showed widening of the defect towards and away from the macula, and three eyes showed widening of the defect towards the macula with deepening of the defect. The remaining two eyes showed a new defect with deepening of the pre-existing defect.

Mean angular widening of the defect towards the macula and away from the macula were $6.4 \pm 4.1^{\circ}$ (range: $1.1-17.1^{\circ}, n=37$ ) and $3.4 \pm 2.1^{\circ}$ (range: $1.1-5.2^{\circ}, n=5$ ), respectively. A new defect appeared in the same quadrant between a pre-existing defect and the macula $(n=4)$, in the area nasal to the pre-existing defect $(n=1)$, and in the vertically opposite quadrant of the preexisting defect $(n=1)$. Mean angles $\alpha$ and $\beta$ of a new defect were $29.8 \pm 35.2^{\circ}$ (range: $5.2-95.8^{\circ}$ ) and $13.6 \pm 5.2^{\circ}$ (range: $5.2-21.6^{\circ}$ ), respectively.

Among 25 eyes that showed deepening of the defect, 16 eyes were initially classified into Grade 1,8 eyes into Grade 2, and 2 eyes into Grade 3. One eye had two separate RNFL defects that showed deepening. In 25 eyes, 17 eyes showed increase in the severity all of which had an increase by one grade. Eleven eyes had sharpening of the defect border, and the location was mostly temporal border $(n=8)$. Sixty-four eyes showed no progression of optic disc cupping. Only one eye showed increased notching at the time when progression of the RNFL defect was detected. Optic disc

haemorrhages were found on the disc stereophotographs taken just before the detection of progression of RNFL defect $(n=2)$, at the time of detection $(n=4)$, and at both $(n=1)$.

Out of the 55 eyes in which automated perimetry were performed after progression of localized RNFL defect, 46 eyes showed no deterioration in the visual field. In the nine eyes that showed progression of visual field defect, four eyes showed progression on the Humphrey central 30-2 threshold visual field, two eyes on the Humphrey Matrix 30-2 visual field, and three eyes converted from the Humphrey Matrix 30-2 visual field defect to the Humphrey central 30-2 threshold visual field defect. Among six eyes that progressed on the Humphrey central 30-2 threshold visual field or on the Humphrey Matrix 30-2 visual field, five eyes showed compatible changes with that of RNFL defect: extension of the preexisting scotoma $(n=2)$ and a new scotoma not within or adjacent to the pre-existing scotoma $(n=3)$. One eye 

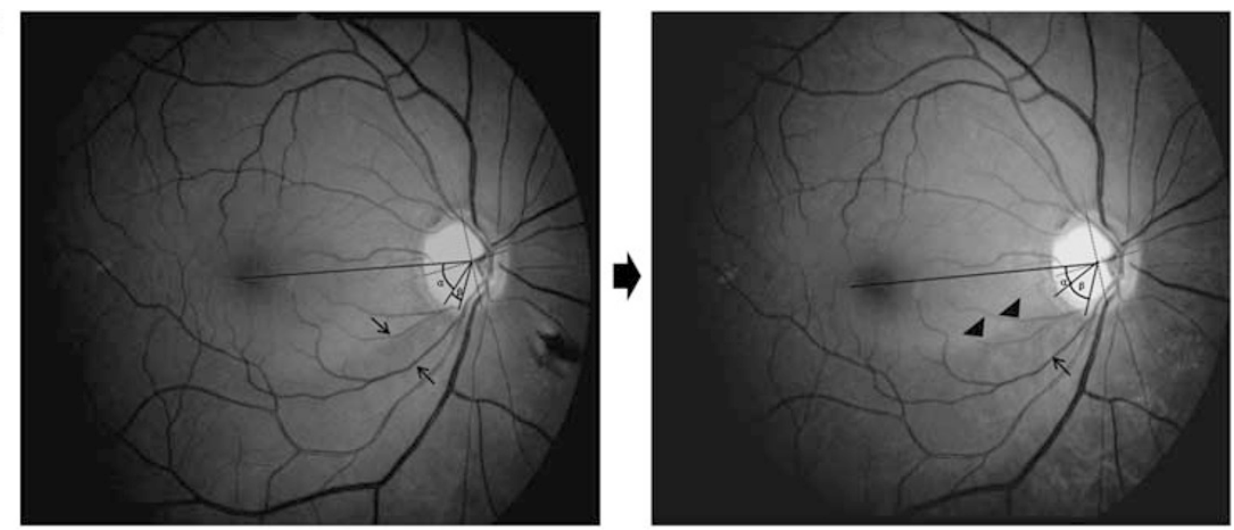

b
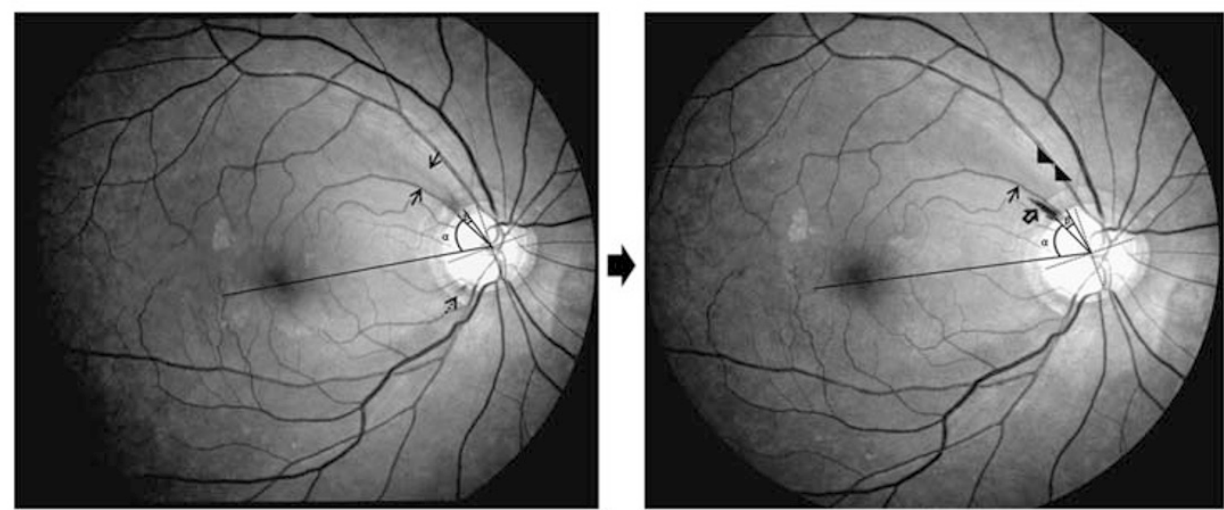

Figure 2 Red-free fundus photographs show widening (arrow heads) of the retinal nerve fibre layer defect (RNFL) (arrows) (a) towards the macula and (b) away from the macula. Angle $\beta$ increased by (a) $12.7^{\circ}$ and (b) $7.2^{\circ}$, respectively. Optic disc haemorrhages were found ( $b$, left) on the opposite side to where there is RNFL defect extension (dashed arrow) and (b, right) on the same side (empty arrow).

showed new scotoma on the Humphrey central 30-2 threshold visual field whereas there was widening of the pre-existing RNFL defect.

\section{Discussion}

In this study, pattern of progression was categorized into four types: widening of the defect towards the macula, deepening of the defect, appearance of a new defect, and widening of the defect away from the macula. To our knowledge, no earlier study had categorized patterns of progression of localized RNFL defect; $56.9 \%$ of eyes (37/65 eyes) showed widening of the defect towards the macula and $66.7 \%$ of new defects (4/6 eyes) appeared between a pre-existing defect and the macula. In addition, sharpening of the defect border mostly developed at the temporal border. As retinal nerve fibres are less detectable in the nasal fundus than in the temporal fundus, RNFL loss should proceed temporally, ${ }^{12}$ especially, sharpening of the defect border is a characteristic feature of deepening of the RNFL defect. This finding concurs with the earlier report suggesting that border of RNFL defect is the most active site of the loss of retinal ganglion cells and their axons. ${ }^{13}$

Pattern of progression of visual field defect was compatible with that of RNFL defect on red-free funds photographs: deepening or extension of the pre-existing scotoma and a new scotoma not within or adjacent to the existing scotoma. ${ }^{11}$ Among six eyes that showed progression on the Humphrey central 30-2 threshold visual field or on the Humphrey Matrix 30-2 visual field, five eyes had compatible pattern of progression with that on red-free fundus photographs. However, this study has limitation of short follow-up period (6.4 \pm 9.0 months) after detection of progression of RNFL defect to correlate visual field change with progression of RNFL defect.

The neural rim of the optic disc is composed of retinal nerve fibres and glaucomatous change should begin in the RNFL and/or optic disc. Badalà et al recently reported that conventional optic disc evaluation by disc stereophotography is superior to automated optic disc and RNFL imaging in detecting early glaucoma. ${ }^{14}$ This study shows that subtle progression of localized RNFL defect can be detected by serial red-free fundus 


\section{a}

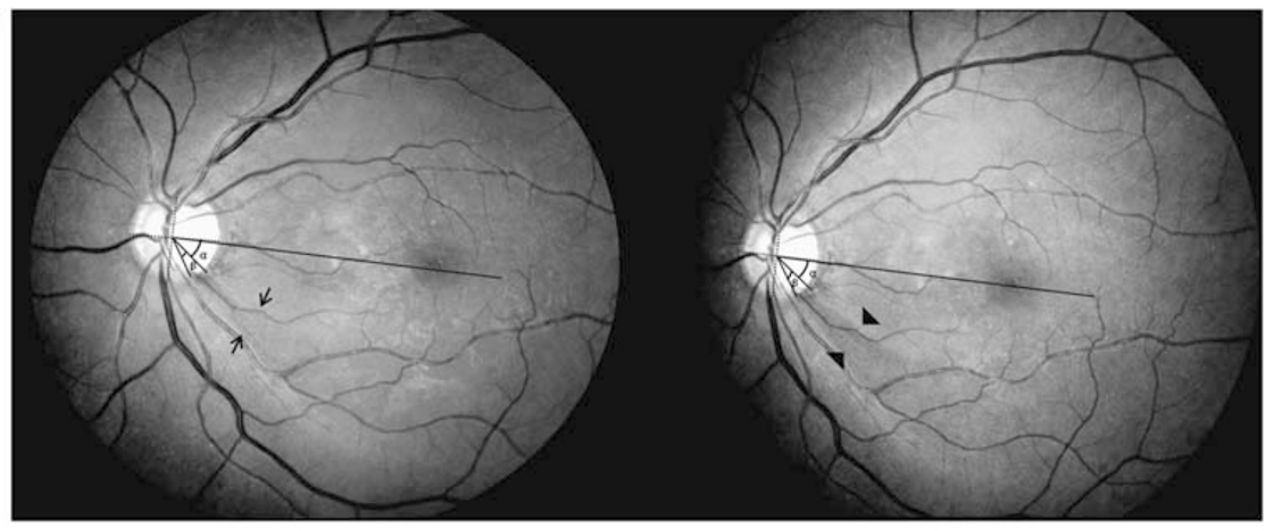

b

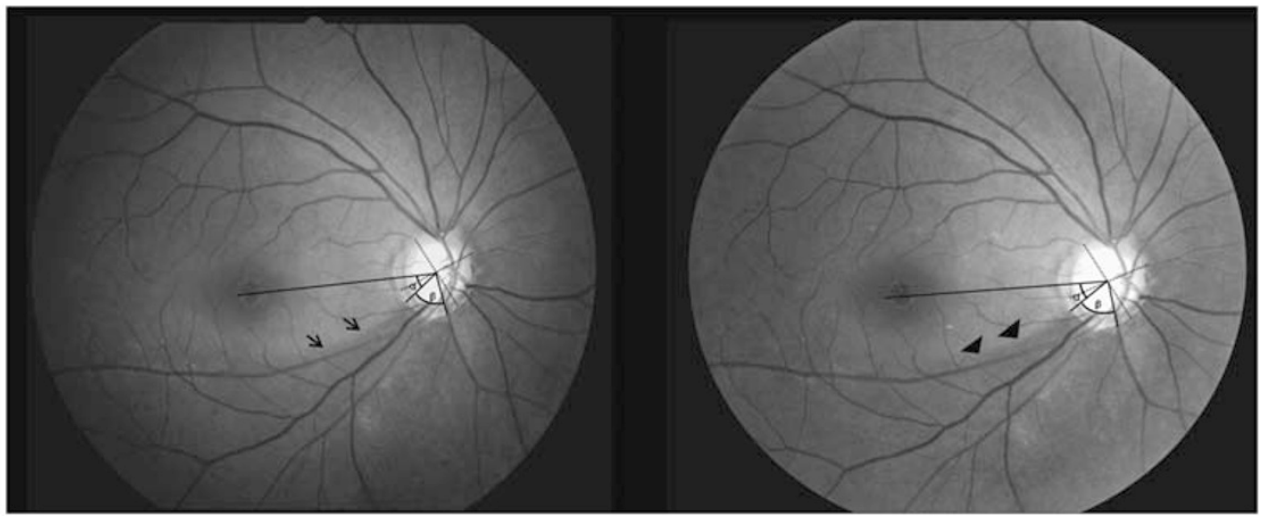

Figure 3 (a) Red-free fundus photographs show deepening (arrow heads) of the localized retinal nerve fibre layer defect (arrows). Severity of the defect increased from Grade 1 to Grade 2. (b) Sharpening of the border (arrow heads) of the pre-existing defect.

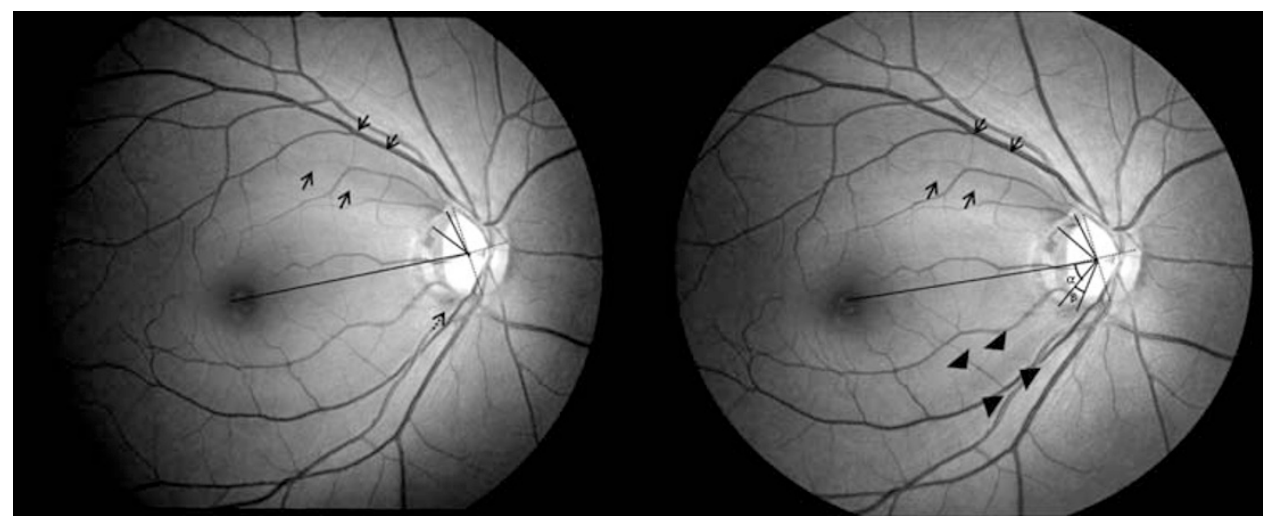

Figure 4 Red-free fundus photograph shows appearance of a new defect (arrow heads). Angles $\alpha$ and $\beta$ of a new defect were $44.5^{\circ}$ and $17.2^{\circ}$, respectively. Optic disc haemorrhage was found (left) before the appearance of the new defect (dashed arrow).

photography, and the changes were hardly detected by disc stereophotography. Therefore, red-free fundus photography should have a role in the early detection of progression of glaucoma in addition to disc stereophotography.

It is sometimes difficult to clearly define the border of diffuse atrophy using red-free fundus photography, and this may lead to problems in categorizing patterns of progression. ${ }^{15}$ Therefore, this study only included localized defects. There remains a question whether deepening of the RNFL defect can be detected objectively. However, this study showed good intra- and inter-observer agreement in grading severity of the defect and determining sharpening of the defect border. 
It should be noted that this study is inherently limited by not applying imaging modalities such as scanning laser tomography that showed higher sensitivity than disc stereophotography in detecting progression of glaucoma. ${ }^{16}$ Further longitudinal studies using imaging modalities are warranted to elucidate the changes.

In conclusion, there were four patterns of progression of localized RNFL defect. In most cases, RNFL loss proceeded temporally.

\section{Acknowledgements}

This work was supported by Grant No. 04-2005-029-0 from the Seoul National University Hospital Research Fund.

\section{Conflict of interest}

The authors declare no conflict of interest.

\section{References}

1 Quigley HA, Reacher M, Katz J, Strahlman E, Gilbert D, Scott R. Quantitative grading of nerve fiber layer photographs. Ophthalmology 1993; 100: 1800-1807.

2 Sommer A, Miller NR, Pollack I, Maumenee AE, George T. The nerve fiber layer in the diagnosis of glaucoma. Arch Ophthalmol 1977; 95: 2149-2156.

3 Quigley HA, Katz J, Derick RJ, Gilbert D, Sommer A. An evaluation of optic disc and nerve fiber layer examinations in monitoring progression of early glaucoma damage. Ophthalmology 1992; 99: 19-28.

4 Giangiacomo A, Garway-Heath D, Caprioli J. Diagnosing glaucoma progression: current practice and promising technologies. Curr Opin Ophthalmol 2006; 17: 153-162.
5 Greenfield DS, Weinreb RN. Role of optic nerve imaging in glaucoma clinical practice and clinical trials. $A m \mathrm{~J}$ Ophthalmol 2008; 145: 598-603.

6 Tuulonen A, Airaksinen PJ. Initial glaucomatous optic disc and retinal nerve fiber layer abnormalities and their progression. Am J Ophthalmol 1991; 111: 485-490.

7 Kim TW, Park UC, Park KH, Kim DM. Ability of Stratus OCT to identify localized retinal nerve fiber layer defects in patients with normal standard automated perimetry results. Invest Ophthalmol Vis Sci 2007; 48: 1635-1641.

8 Hoyt WF, Frisen L, Newman NM. Fundoscopy of nerve fiber layer defects in glaucoma. Invest Ophthalmol Vis Sci 1973; 12: 814-829.

9 Woo SJ, Park KH, Kim DM. Comparison of localised nerve fibre layer defects in normal tension glaucoma and primary open angle glaucoma. Br J Ophthalmol 2003; 87: 695-698.

10 Schulzer M. Errors in the diagnosis of visual field progression in normal-tension glaucoma. Ophthalmology 1994; 101: 1589-1594.

11 Boden C, Blumenthal EZ, Pascual J, McEwan G, Weinreb RN, Medeiros F et al. Patterns of glaucomatous visual field progression identified by three progression criteria. Am J Ophthalmol 2004; 138: 1029-1936.

12 Jonas JB, Schiro D. Localised wedge shaped defects of the retinal nerve fiber layer in glaucoma. Br J Ophthalmol 1994; 78: 285-290.

13 Ishida K, Yamamoto T, Sugiyama K, Kitazawa Y. Disk hemorrhage is a significantly negative prognostic factor in normal-tension glaucoma. Am J Ophthalmol 2000; 129: 707-714.

14 Badalà F, Nouri-Mahdavi K, Raoof DA, Leeprechanon N, Law SK, Caprioli J. Optic disk and nerve fiber layer imaging to detect glaucoma. Am J Ophthalmol 2007; 144: 724-732.

15 Airaksinen PJ, Tuulonen A, Werner EB. Clinical evaluation of the optic disc and retinal nerve fiber layer. In: Ritch R, Shields MB, Krupin T (eds). The Glaucomas, Vol 1. Mosby: St Louis, MO, 1996, pp 617-657.

16 Kourkoutas D, Buys YM, Flanagan JG, Hatch WV, Balian C, Trope GE. Comparison of glaucoma progression evaluated with Heidelberg retina tomograph II vs optic nerve head stereophotographs. Can J Ophthalmol 2007; 42: 82-88. 